Annals of Plant Sciences

\title{
Two new additions to the flora of Telangana, India.
}

\section{J. Swamy* and E. Venkatesham}

Botanical Survey of India, Deccan Regional Centre, Hyderabad-500048, Telangana, India.

Department of Botany, Osmania University, Hyderabad-500007, Telangana, India.

Received: 05-12-2017; Accepted: 17-12-2017

Abstract: Ipomoea triloba L. and Merremia dissecta (Jacq.) Hallier f. are reported here as an addition to the flora of Telangana. Detailed descriptions and photo plates are provided to facilitate its easy identification.

Keywords: Convolvulaceae, Ipomoea, Merremia, new additions, Rangareddy, Telangana

\section{Introduction}

While exploring the weed flora of Greater Hyderabad, the authors came across and collected two species of Convolvulaceae vir., Ipomoea triloba $\mathrm{L}$. and Merremia dissecta (Jacq.) Hallier f. Perusal of literatures revealed that, these two species has so far been not reported from Telangana (1). Hence, both the species are reported here as new additions to the flora of Telangana.

\section{Taxonomic Treatment}

Ipomoea triloba L. Sp. Pl. 1:161.1753; Magesh et al. in Zoo's Print 5: 24.2012. Ipomeoa blancoi Choisy in DC., Prodr. 9: 389.1845.

Annual twining herbs; stems twining or sometimes prostrate, 1-3 m long, glabrous or sparsely hairy at the nodes. Leaves broadly ovate to orbicular in outline, rarely narrower, 3-9 × 2-8-8.5 cm, margin entire or coarsely dentate to more or less deeply 3lobed, cordate at base, basal lobes rounded or angular, acute at apex, glabrous; petiole slender, 2.5$8 \mathrm{~cm}$, glabrous. Inflorescences axillary, dense umbellate cymes, one to several flowered; peduncle shorter to longer than the petiole, $2-10 \mathrm{~cm}$, glabrous, angular, minutely verrucose towards the apex; branches of the cyme very short, 7-10 mm long. Pedicels more or less angular, minutely verrucose, glabrous, 5-7.5 mm long. Bracts minute, oblong-lanceolate, $1.5 \mathrm{~mm}$ long, slightly recurved at apex. Sepals slightly unequal, 8-10 × 3-4 mm, the outer ones a slightly shorter, narrowly oblong, fimbriate along margin, obtuse or acute, mucronulate at apex, sparsely pilose on the back; inner sepals somewhat broader, elliptic-oblong, acute, mucronulate, glabrous or sparsely hairy. Corolla funnel-shaped, $18-20 \mathrm{~mm}$ long, glabrous, pink or pale purple, sometimes with darker centre; tube c. $15 \mathrm{~mm}$ long, 5-lobed; lobes 4.5-5 × 4-4.5 $\mathrm{mm}$, obtuse, mucronulate at apex. Stamens included c. $10 \mathrm{~mm}$ long; filaments, unequal, 7-9 $\mathrm{mm}$ long, hairy at the base; anthers $1-1.2 \times 0.5 \mathrm{~mm}$ long. Pistil included, c. $11 \mathrm{~mm}$ long; ovary $1.5 \times 1.2 \mathrm{~mm}$, hairy; style $9 \mathrm{~mm}$ long; stigma 2-lobed, $0.7 \times 1 \mathrm{~mm}$. Capsule sub-globular, 5-6 $\times$ 7-8 mm, bristly hairy, apiculate, 2-celled, 4-valved, 4-seeded; seeds dark brown, 3-5 × 3-4 mm long, glabrous (Fig.1).

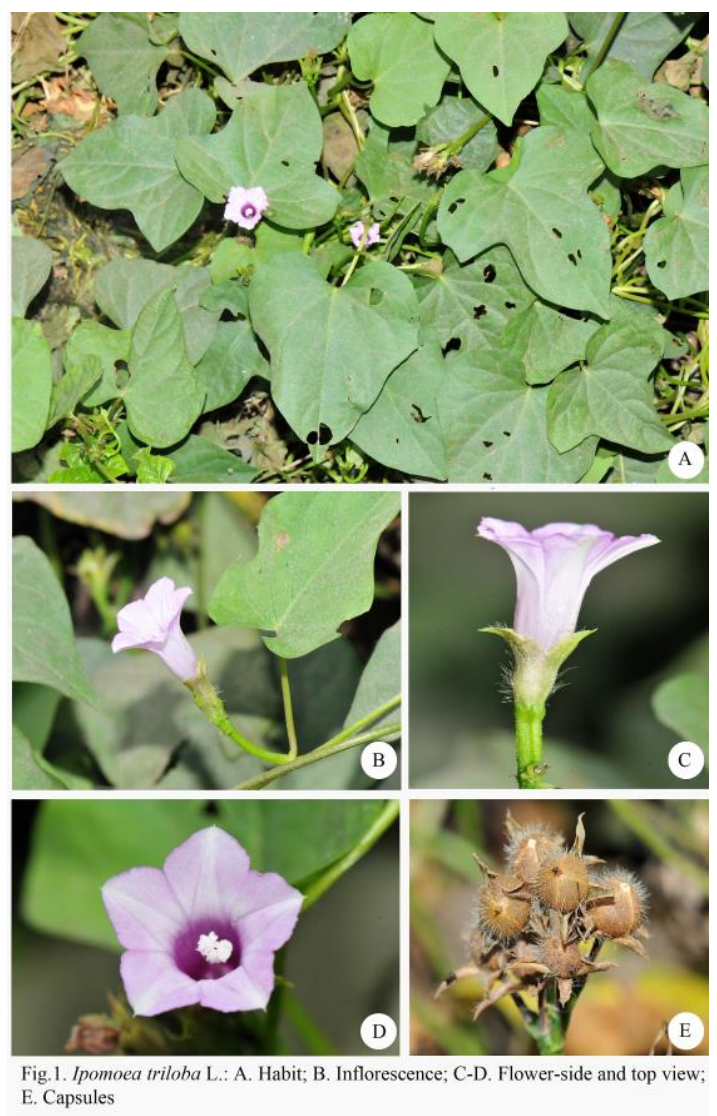

Flowering \& Fruiting: October-January.

Habitat: Common along waysides, waste places and hedges.

Distribution: World: Native of tropical America, now a circumtropical weed. India: Gujarat,

\section{*Corresponding Author:}

J. Swamy,

Botanical Survey of India,

Deccan Regional Centre,

Hyderabad-500048, India.

E-mail: swamy.2706@gmail.com 
Jharkhand, Karnataka, Kerala, Maharashtra, Rajasthan, Tamil Nadu, Uttar Pradesh, West Bengal and now from Telangana.

Specimen examined: Telangana, Rangareddy district, Near Herbal Garden, on the way to Aramghar, $17.312964^{\circ} \mathrm{N}, 78.423869^{\circ} \mathrm{E}, 539$ m.a.s.l., 4th December, 2017, J. Swamy (4342), (BSID).

Merremia dissecta (Jacq.) Hallier f., Bot. Jahrb. Syst. 16 (4-5): 552. 1893; Gamble, Fl. Madras 928. 1923; Convolvulus dissectus Jacq., Obs. Bot. 2: 4. 28. 1767. Ipomoea sinuata Ortega in Nov. Rar. Pl. Descr. Dec. 84.1798; Hook. f., Fl. Brit. India 4: 214. 1883.

Herbaceous twiner; stems slender, terete, striate, patently hirsute, glabrescent. Leaves alternate, palmately divided nearly to the base, with $5-7$ lanceolate, mucronulate, sinuate, coarsely dentate to irregularly pinnately lobed segments, glabrous, the middle segment $4-7.5 \mathrm{~cm}$ long, $1-2.5 \mathrm{~cm}$ broad, the lateral ones and especially the basal ones smaller; petiole up to $7 \mathrm{~cm}$ long, patently hirsute. Inflorescence axillary, 1-to few-flowered, cymose; peduncles 5-10 cm long, patently hirsute, glabrescent in the upper portion hirsute; pedicels $1.5-2.5 \mathrm{~cm}$ long, thickened above, glabrous, minutely verrucose at the top. Flower-buds 1.5$2.5 \mathrm{~cm}$ long, narrow-ovoid, acute at apex. Sepals sub-equal, 2-2.5 cm long, ovate or oblong, mucronulate at apex, glabrous, herbaceous with narrow scarious margin, afterwards enlarged and coriaceous in fruit. Corolla $3-4 \mathrm{~cm}$ long, funnelshaped, white, with a rose or purple throat, the limb with 5 distinct bands. Stamens 5, unequal, included; filaments $5-7 \mathrm{~mm}$ long, filiform, villous at base, glabrous towards apex; anthers up to $5 \mathrm{~mm}$ long, spirally twisted above the half. Disk cup-shaped. Pistil c. $2 \mathrm{~cm}$ long; ovary c. 1.5 x $1.5 \mathrm{~mm}$ long glabrous, 2-celled; style c. $8 \mathrm{~mm}$ long; stigma bilobed, globose. Capsule globose, $1-2 \mathrm{~cm}$ in diameter, glabrous, 4-valved, 4-seeded; seeds c. 1.2 x $0.8 \mathrm{~mm}$, black, glabrous. (Fig. 2).

Flowering \& Fruiting: August-January.

Habitat: Occasionally found in waste places and hedges.

Distribution: World: Probably indigenous to America (Southern United States, Central America, West Indies, South America to Argentina and Urugua), becoming naturalized in Africa (Upper Guinea, Cordofan and Seychelles), Australia (Queensland), Asia (China, India, Indonesia, Malaysia, Myanmar, Pakistan, Sri Lanka, Thailand, Taiwan and Saudi Arabia) as a garden escape (2). India: Throughout in warm places.
Specimen examined: Telangana, Rangareddy district, Professor Jaya Shankar Telangana State Agricultural University, $17.33777778^{\circ} \mathrm{N}$, 78.41444440 E, 530 m.a.s.l., 11 th September, 2016, J. Swamy (4339), (BSID).
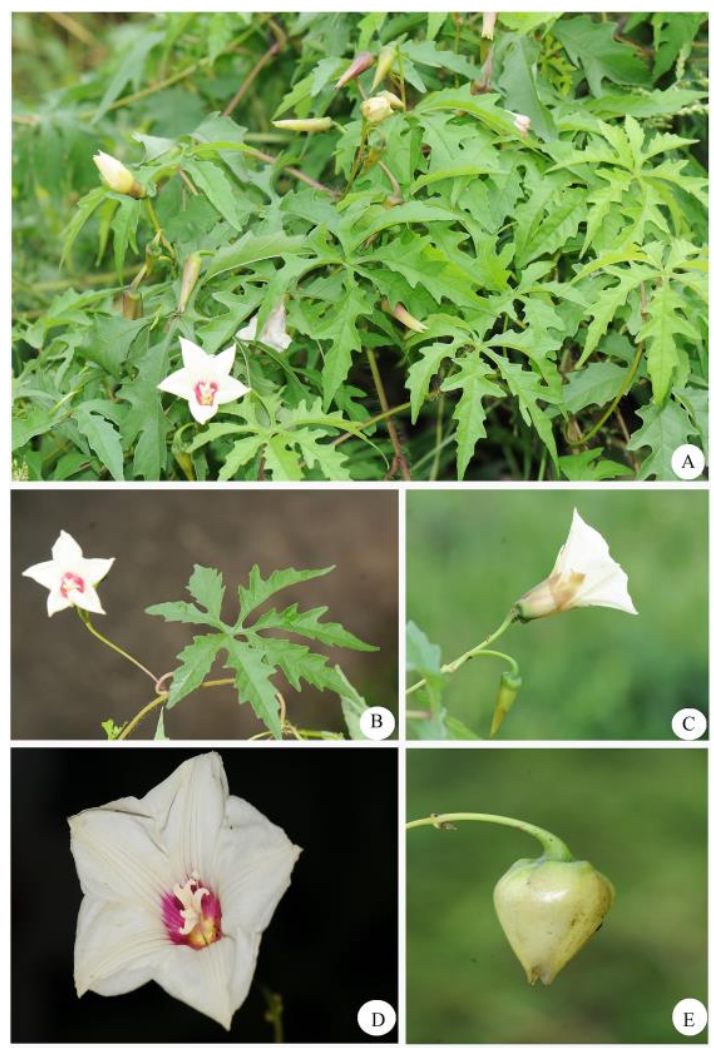

Fig. 2. Merremia dissecta (Jacq.) Hallier f. : A. Habit; B-C.Inflorescence; D. Flower; E. Capsule

\section{Acknowledgements}

Authors are thankful to Director, Botanical Survey of India, Kolkata and Dr. L. Rasingam, Scientist Incharge, Botanical Survey of India, Deccan Regional Centre, Hyderabad for facilities.

\section{References}

1. Pullaiah T, Flora of Telangana, the 29th State of India. Regency Publications, New Delhi 2 (2015): 651-669 Print.

2. Ooststroom SJ van. The Convolvulaceae of Malaysia. Blumea 3.2 (1939): 352-371 Print.

\section{Cite this article as:}

Swamy J. and E. Venkatesham. Two new additions to the flora of Telangana, India. Annals of Plant Sciences 7.1 (2018) pp. 1952-1953.

d o $\mathrm{http://dx.doi.org/10.21746/aps.2018.7.1.6}$ 